\title{
Is a Potassium-Competitive Acid Blocker Truly Superior to Proton Pump Inhibitors in Terms of Helicobacter pylori Eradication?
}

\author{
Soichiro Sue and Shin Maeda \\ Department of Gastroenterology, Yokohama City University Graduate School of Medicine, Yokohama, Japan
}

\section{Article Info}

Received July 31, 2020

Revised November 17, 2020

Accepted December 1, 2020

Corresponding Author

\section{Shin Maeda}

ORCID https://orcid.org/0000-0002-0246-1594

E-mail shinmaeda2-gi@umin.ac.jp

\begin{abstract}
Vonoprazan (VPZ), a new potassium-competitive acid blocker, has been approved and used for Helicobacter pylori eradication in Japan. To date, many studies, as well as several systematic reviews and meta-analyses (MAs), have compared VPZ-based 7-day triple therapy with proton pump inhibitor (PPI)-based therapy. An MA of randomized controlled trials (RCTs) comparing first-line VPZ- with PPI-based triple therapy, the latter featuring amoxicillin (AMPC) and clarithromycin (CAM), found that approximately $30 \%$ of patients hosted CAM-resistant $H$. pylori; however, the reliability was poor because of high heterogeneity and a risk of selection bias. VPZ-based triple therapy is superior to PPI-based triple therapy for patients with CAM-resistant $H$. pylori, but not for those with CAM-susceptible H. pylori. An MA of non-RCTs found that second-line VPZbased triple therapies were slightly $(\sim 2.6 \%)$ better than PPI-based triple therapies (with AMPC and metronidazole). However, the reliability of that MA was also low because of selection bias, confounding variables and a risk of publication bias; in addition, it is difficult to generalize the results because of a lack of data on antibiotic resistance. VPZ-based triple therapy (involving AMPC and sitafloxacin) was more effective than PPI-based triple therapy in a third-line setting, but a confirmatory RCT is needed. Non-RCT studies indicated that VPZ-based triple therapy involving CAM and metronidazole may be promising. Any further RCTs must explore the antibiotic-resistance status when evaluating the possible superiority of a potassium-competitive acid blocker. (Gut Liver 2021;15:799-810)
\end{abstract}

Key Words: Potassium-competitive acid blocker; Proton pump inhibitors; Helicobacter pylori; Treatment outcome; Drug resistance, microbial

\section{INTRODUCTION}

Helicobacter pylori-induced signaling pathways contribute to the development of gastric carcinogenesis. ${ }^{1} \mathrm{~A}$ systematic review (SR) and meta-analysis (MA) found that H. pylori eradication reduced the incidence and mortality rates of gastric cancer. ${ }^{2}$ Many clinical trials have assessed the efficacy and safety of $H$. pylori eradication regimens. ${ }^{3}$ An intention-to-treat (ITT) cure rate that is "excellent" ( $95 \%$ to $100 \%$ ) is considered optimal, and a "good" cure rate $(90 \%$ to $95 \%)$ is considered acceptable. ${ }^{4}$ It is important to increase the gastric $\mathrm{pH} ; \mathrm{H}$. pylori then enters an antibiotic-susceptible replicative state. ${ }^{5}$ Several MAs have shown that high-dose proton pump inhibitors (PPIs) enhance eradication.$^{6-8}$ Vonoprazan (VPZ) is a new potassiumcompetitive acid blocker (P-CAB) approved in 2015 for H. pylori eradication in Japan. ${ }^{9}$ Since that time, several SRs and MAs comparing VPZ- and PPI-based therapies have appeared, ${ }^{10-12}$ but the same studies were reviewed among several of the MAs. Furthermore, few randomized controlled trials (RCTs) have been performed, ${ }^{13}$ and many studies lacked data on antibiotic resistance. Here, we focus on study overlap and design and antibiotic resistance data. We pose the question: is $\mathrm{P}-\mathrm{CAB}$ really superior to a PPI in terms of $H$. pylori eradication? 


\section{MECHANISM AND CLINICAL INDICATION OF P-CAB}

\section{Mechanism of P-CAB action in patients with various lesions}

VPZ is a new P-CAB (other P-CABs include SCH28080) that inhibits $\mathrm{H}+/ \mathrm{K}+$ ATPases in a manner described as rapid (the intragastric $\mathrm{pH}$ increased to over 4.0 within 4 hours ${ }^{14}$ ), strong (the intragastric $\mathrm{pH}$ increased to over 5 and was maintained for $99 \%$ of the time when VPZ [20 $\mathrm{mg}$ ] was given twice daily ${ }^{15}$ ), or stable (not affected by the CP2C19 genotype $\left.{ }^{14,16}\right)$. VPZ was the second P-CAB to be approved worldwide (revaprazan was approved first, in South Korea).

At $\mathrm{pH}>5, H$. pylori enters the growth phase. Clarithromycin (CAM) inhibits protein synthesis during growth, and amoxicillin (AMPC) inhibits cell wall biosynthesis; metronidazole (MNZ) targets DNA synthesis and acts during both the growth and stationary phases. ${ }^{17}$ Thus, CAM and AMPC function at $\mathrm{pH}>5$, whereas $\mathrm{MNZ}$ is $\mathrm{pH}$ independent.

$\mathrm{SCH} 28080$ is the prototype $\mathrm{P}-\mathrm{CAB}$ that was developed in the 1980s. This drug is short-acting and was never approved. Linaprazan was found to be as effective as esomeprazole $(40 \mathrm{mg})$ in patients with non-erosive reflux disease; however, its clinical development was later suspended. ${ }^{18}$ Revaprazan (a P-CAB) was approved in South Korea in 2005 for the treatment of gastroduodenal ulcers and gastritis. However, endoscopic submucosal dissection revealed that the drug was no more efficacious than $20 \mathrm{mg}$ rabeprazole for treating ulcers. ${ }^{19}$ In 2018, a new P-CAB, tegoprazan, was approved for $H$. pylori eradication in South Korea. Tegoprazan was not inferior to lansoprazole when used to treat gastric ulcers ${ }^{20}$ and non-inferior to esomeprazole in patients with erosive esophagitis. ${ }^{21}$ However, no data on $H$. pylori eradication have been published. Tegoprazan may be valuable in this context.

\section{P-CAB based data: mainly with VPZ, in Japan, and with triple therapy}

P-CAB based data are mainly with VPZ based and in Japanese population. First-line VPZ based regimens compared to PPI based (Table 1), and second-line VPZ based regimens compared to PPI based (Table 2) are studies with Japanese population. In these studies, 7-day triple therapies are used. In Japan, 7-day first-line triple therapy consisting of VPZ or a PPI, AMPC, and CAM and 7-day secondline triple therapy consisting of VPZ or a PPI, AMPC, and MNZ are covered by national insurance. Esomeprazole, rabeprazole, lansoprazole, or omeprazole serves as the PPI. The approved doses are VPZ $20 \mathrm{mg}$ bid (twice a day; 40 $\mathrm{mg}$ /day), esomeprazole $20 \mathrm{mg}$ bid (40 mg/day), rabeprazole $10 \mathrm{mg}$ bid ( $20 \mathrm{mg} /$ day), omeprazole $20 \mathrm{mg}$ bid (40 $\mathrm{mg}$ /day), AMPC $750 \mathrm{mg}$ bid (1,500 mg/day), CAM $200 \mathrm{mg}$ or $400 \mathrm{mg}$ bid ( $400 \mathrm{mg} /$ day or $800 \mathrm{mg} /$ day), and MNZ 250 $\mathrm{mg}$ bid (500 mg/day).

\section{Antibiotic resistance background in a Japanese population}

Studies reviewed in this article are based on a Japanese population, so the Japanese $H$. pylori antibiotic-resistance status is important to understanding the setting and limitation of this review. One MA found that CAM resistance reduced the eradication rate by $55 \%$ (95\% confidence interval [CI], 33 to 78), and MNZ resistance reduced the rate by $37.7 \%$ (95\% CI, 29.6 to 45.7 ); CAM/MNZ resistance is the principal cause of eradication failure. ${ }^{22}$ Whereas the MNZresistance rate remains low, ${ }^{23}$ the CAM-resistance rate has increased, from $23.7 \%(56 / 236)$ in $2017^{24}$ to $27.9 \%$ (41/147) in $2018 .{ }^{25}$ Table 1 lists the available data on antibiotic resistance. The CAM-resistance rates were $35.5 \%(215 / 605)$ in $2016,{ }^{9} 39.5 \%$ (45/114) in $2016,{ }^{26} 34.7 \%$ (143/412) in $2016,{ }^{27}$ $42.7 \%(123 / 288)$ in $2019,{ }^{28}$ and $25.6 \%(42 / 164)$ in $2020 .{ }^{29}$ The data differed according to the lesion type evaluated; the average was $33.8 \%(665 / 1,996)$ (95\% CI, 31.7 to 36.0$)$. The CAM-resistance rate exceeds $15 \%$ in Japan, which is thus a high-CAM-resistance area. As shown in Table 2, MNZ-resistance data are scarce. Horie et al. ${ }^{23}$ reported that the MNZ-resistance rate was less than 5\% from 2005 to 2018. The AMPC-resistance rate is generally very low in Japan. In 2020, Suzuki reported that the minimal inhibitory concentration of AMPC was $<0.03 \mu \mathrm{g} / \mathrm{mL}$ in $93.6 \%$ (306/327) of subjects, $0.03 \mu \mathrm{g} / \mathrm{mL}$ in $5.2 \%$ (17/327) of subjects, and $0.06 \mu \mathrm{g} / \mathrm{mL}$ in $1.2 \%(4 / 327)$ of subjects. ${ }^{29}$ In summary, Japanese population-based eradication studies have found high rates ( 33\%) of CAM resistance, low rates $(<5 \%)$ of MNZ resistance, and very low rates of AMPC resistance. This antibiotic resistance setting is the main limitation of this review in generalizing to clinical settings outside of Japan.

\section{VPZ-COMPARED WITH PPI-BASED FIRST-LINE TRIPLE THERAPY CONSISTING OF AMPC AND CAM}

As mentioned above, we ask: "is P-CAB really superior to a PPI in terms of $H$. pylori eradication?" in the context of first-line VPZ-based 7-day triple therapy consisting of AMPC and CAM. As shown in Table 1, many relevant studies have appeared. 


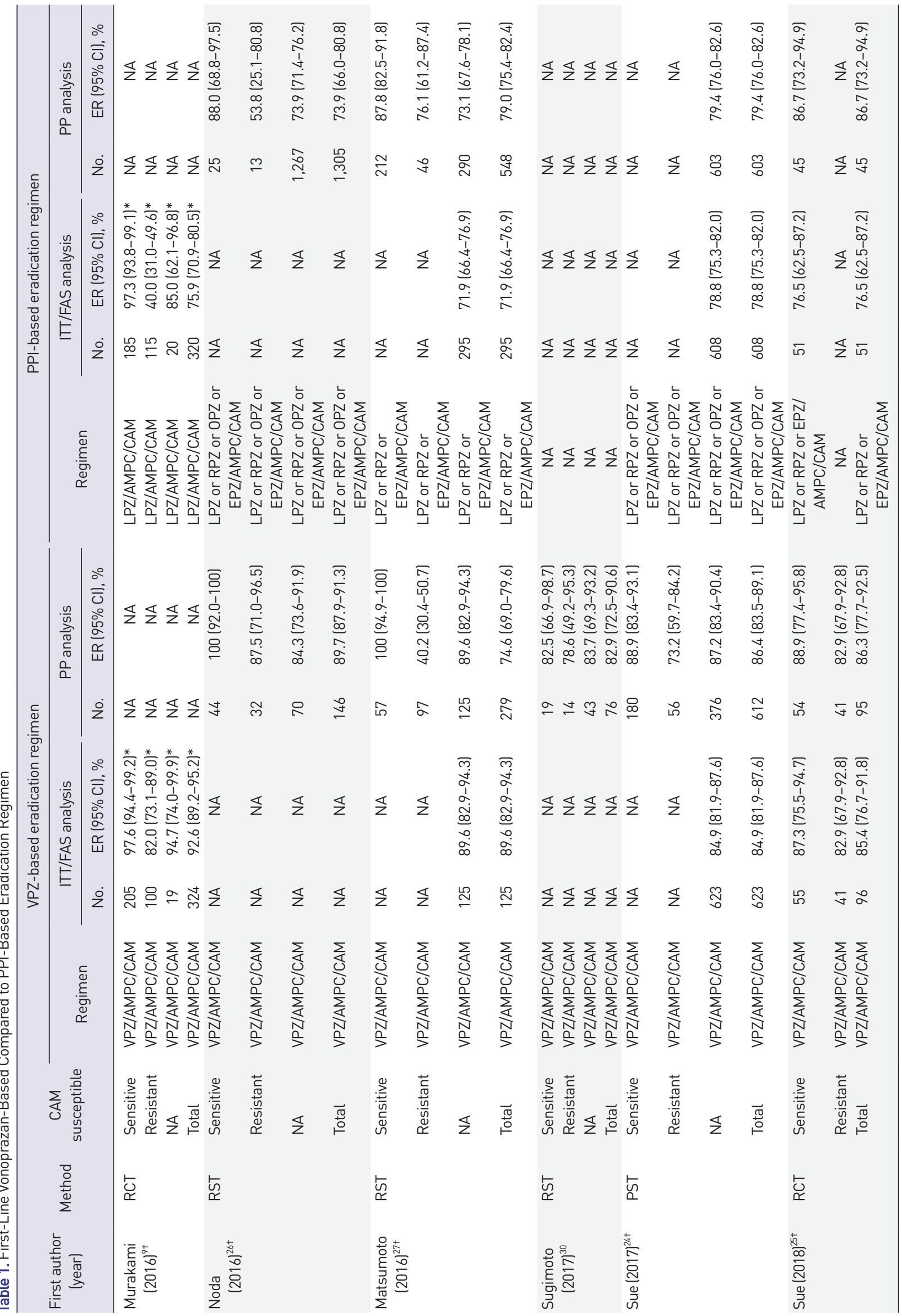




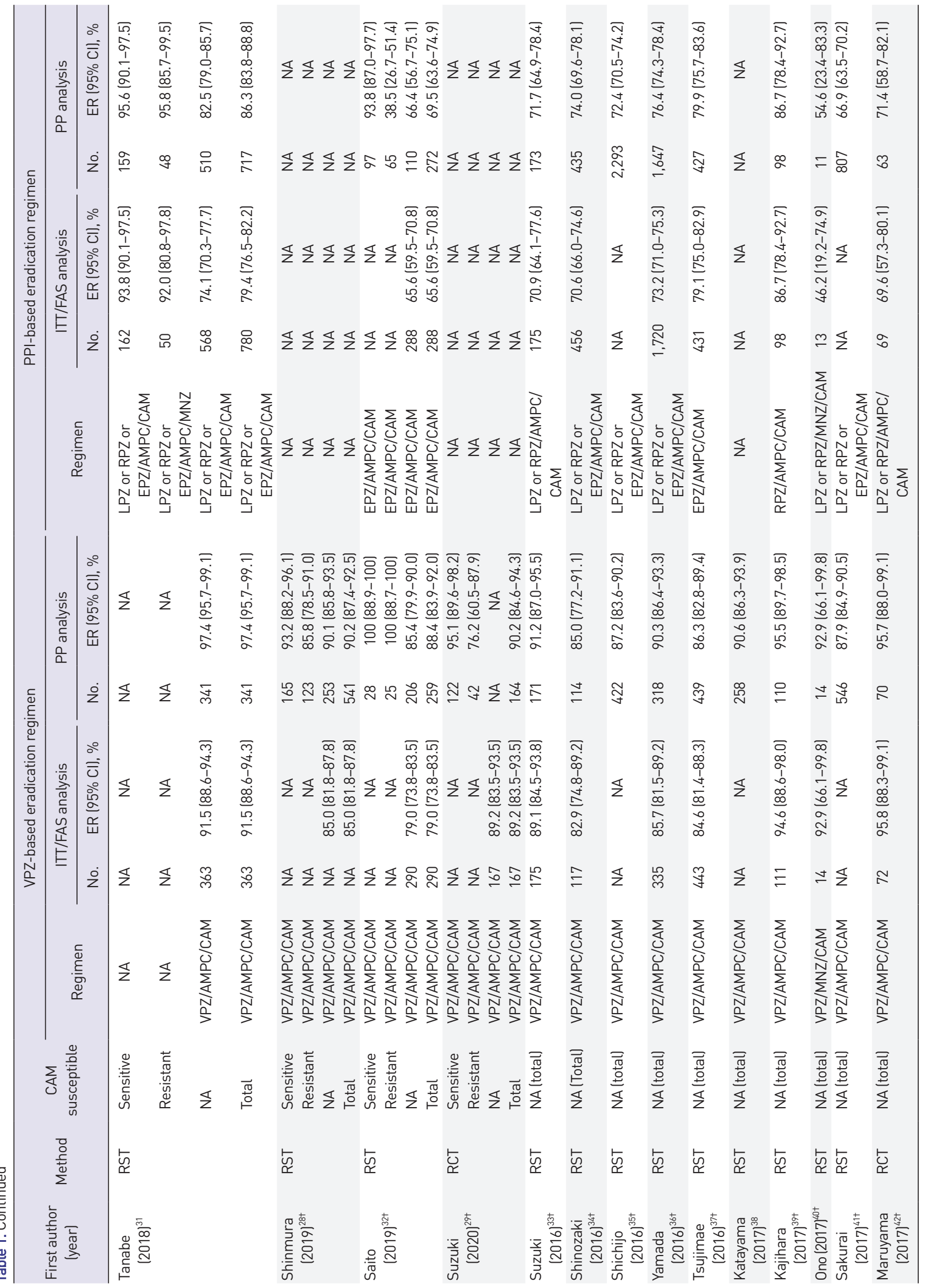




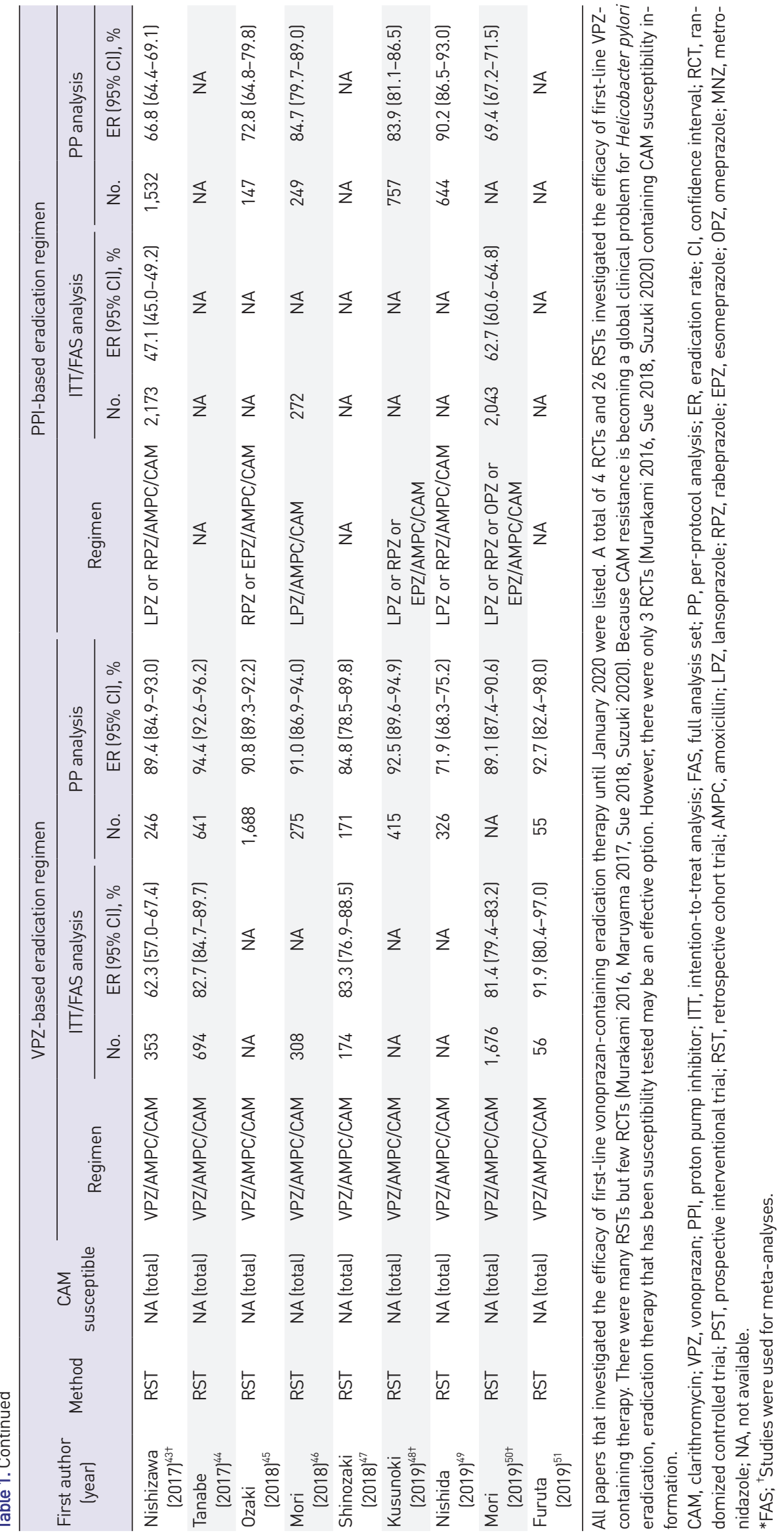




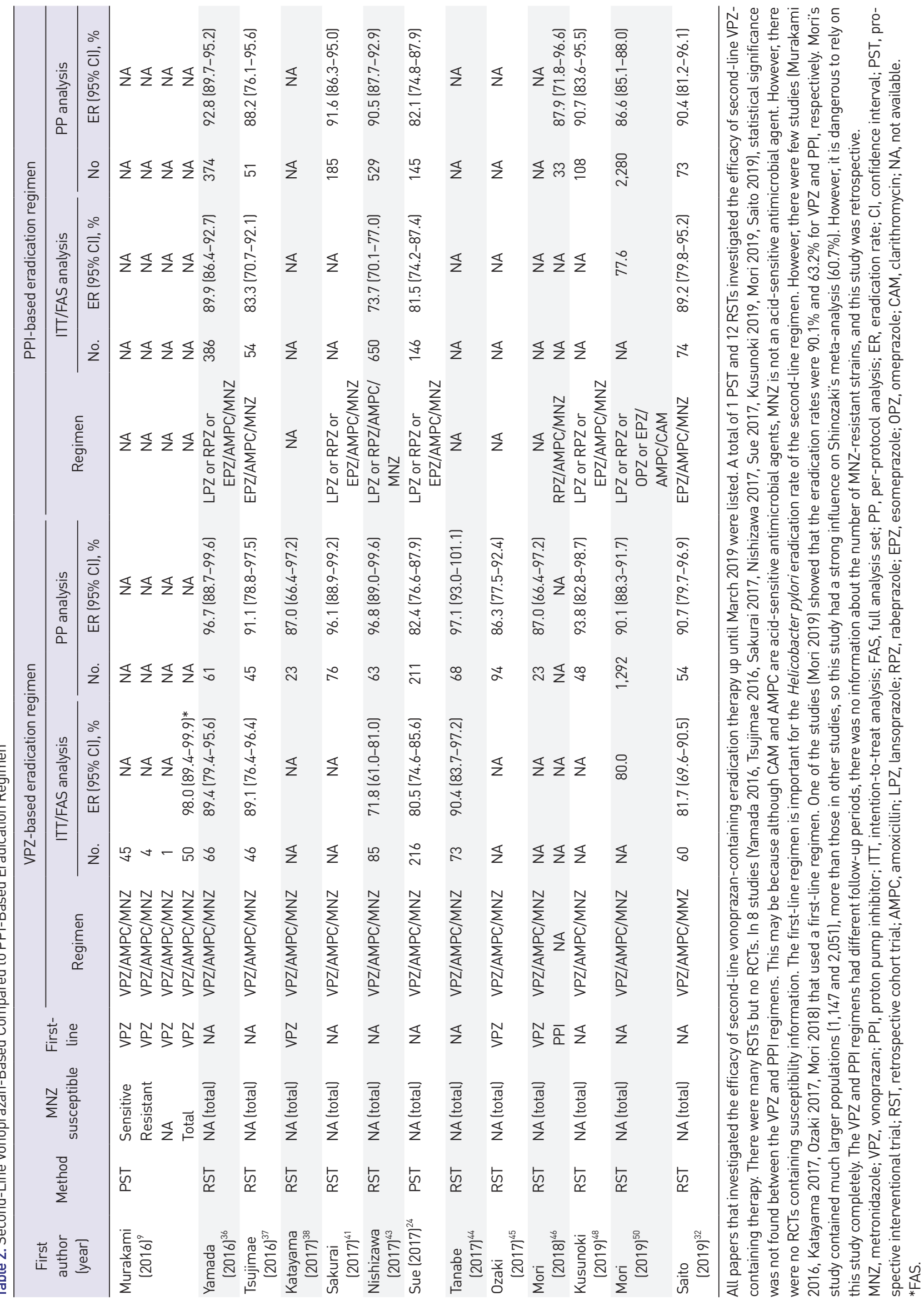




\section{The need for CAM-resistance data}

The CAM-resistance status is very important when exploring whether first-line 7-day triple therapy consisting of VPZ, AMPC, and CAM are superior to PPI-based regimens. Attempts to generalize results in the absence of CAM- and AMPC-resistance data ${ }^{33-51}$ are both difficult and misleading. Generalization may be possible in very limited circumstances only (the trial sites and lesions are identical). The CAM-resistance rate is increasing in Japan, and antibiotic-resistance rates vary by lesion. ${ }^{9,24-32}$ Generalization to other countries is even less appropriate.

\section{MAs of RCTs}

MAs of RCTs evaluated high-quality evidence. In 2019, Lyu et al. ${ }^{13}$ concluded that VPZ-based triple therapies were superior to PPI-based triple therapies based on ITT analysis $(91.4 \%$ [95\% CI, 88.5 to 93.8 ] vs $74.8 \%$ [95\% CI, 70.5 to 78.8$]$ ) and per-protocol (PP) analysis (92.6\% [95\% CI, 89.8 to 94.9 ] vs $76.4 \%$ [95\% CI, 72.1 to 80.3 ], respectively). Three RCTs were analyzed, ${ }^{9,25,42}$ of those, one ${ }^{42}$ had a risk of bias because of allocation concealment, as randomization was based on personal medical record numbers (odd or even). A risk of selection bias was involved during the assignment of 141 of 1,482 chronic gastritis cases (72 and 69 to the VPZ- and PPI-based treatments, respectively). In another of the three RCTs, all subjects were CAM susceptible. $^{25}$ The final RCT evaluated was a phase III trial conducted prior to approval of a new drug in Japan (performed before VPZ approval); ${ }^{9}$ such trials usually exhibit selection bias. Heterogeneity was moderate in the ITT analysis $\left(\mathrm{I}^{2}=46 \%\right)$ and high in the PP analysis $\left(\mathrm{I}^{2}=61 \%\right)$, indicating that the MA was not reliable. If an MA is generalizable, information on antibiotic resistance is important. Two of the above RCTs contained such data, ${ }^{9,25}$ whereas the third did not. ${ }^{42}$ Of those receiving VPZ-based therapy, 259 were CAM susceptible, 100 were CAM resistant, and 73 were labeled "not applicable;" of those receiving PPI-based therapy, 230 were CAM susceptible, 115 were CAM resistant, and 64 were labeled "not applicable." The CAM-resistance rates were approximately $27.9 \%$ and $33.3 \%$ among those receiving VPZ-based and PPI-based therapies, respectively; thus, it might be possible to generalize the result to populations containing approximately 30\% CAM-resistant subjects. However, the CAM-resistance rate was lower in the VPZ-treated than PPI-treated group (27.9\% vs 33.3\%), biasing the results. We thus focused on the treatment efficacy in CAM-susceptible and -resistant subjects in sections 3.4 and 3.5 below. In summary, one MA of RCTs indicated that VPZ-based therapy may be superior to PPIbased therapy in populations exhibiting approximately $30 \%$ CAM resistance, but the reliability of that MA was low given the high heterogeneity and risk of selection bias (lack of allocation concealment).

\section{MAs of non-RCTs}

Table 1 shows that many non-RCTs have been performed, but retrospective cohort studies lacking information on antibiotic resistance are misleading, as the CAMresistance rates might have differed. Several MAs lack antibiotic-resistance data and are as misleading as single retrospective cohort studies lacking this information. The MA by Dong et al. $(2017)^{10}$ discussed two RCTs, ${ }^{9,42}$ and 12 non-RCTs. ${ }^{24,26,27,33-37,39-41,43}$ One study ${ }^{33}$ performed propensity score matching in the absence of antibiotic-resistance data. Another ${ }^{40}$ featured triple therapy consisting of CAM, MNZ or MNZ, and sitafloxacin (STFX; 88 of 13,495 cases); we discuss this in the sixth chapter. That retrospective study lacking antibiotic-resistance data was misleading. The eradication rate was $85.1 \%$ in VPZ-treated patients versus $68.0 \%$ in PPI-treated patients $(p<0.00001)$ in the ITT analysis and $89.0 \%$ versus $74.2 \%$ in the PP analysis $(\mathrm{p}<0.00001) .{ }^{10}$ Heterogeneity was high in the non-RCT analysis $\left(\mathrm{I}^{2}=65 \%\right)$ and low-to-moderate in the RCT analysis $\left(\mathrm{I}^{2}=26 \%\right)$, suggesting that the non-RCT data are unreliable. Jung et al. $(2017)^{11}$ discussed one $\mathrm{RCT}^{9}$ and nine non$\mathrm{RCTs}^{26,27,33-36,39,41}$ Heterogeneity was high in the non-RCT analysis $\left(\mathrm{I}^{2}=72 \%\right)$, suggesting that the MA was as unreliable as that by Dong et al.

The MA by Li et al. ${ }^{12}$ discussed two RCTs, ${ }^{9,25}$ and three non-RCTs ${ }^{24,26,27}$ with a focus on CAM-resistant and CAMsusceptible subjects separately. We discuss that MA in the next section.

No MA presented a funnel plot; we suspect that publication bias explains many of the differences between RCTs and non-RCTs. Many retrospective studies have been presented in Japanese conferences in Japanese, of which few are published. Most studies are neither prospective nor registered. Well-designed, registered, prospective studies with pre-planned analysis methods would reduce publication bias. In summary, MAs that include non-RCTs are unreliable given their high heterogeneity and publication bias, and it is difficult to generalize the results when antibioticresistance data are lacking.

\section{CAM-resistant subjects}

In 2017, Dong et al. ${ }^{10}$ published an MA of CAM-resistant subgroups given first-line triple therapy consisting of AMPC and CAM. The eradication rate was $81.5 \%(95 \%$ CI, 75.0 to 86.9 ) in the VPZ-based group versus $40.9 \%$ (95\% CI, 34.4 to 47.6 ) in the PPI-based group (odds ratio [OR], 5.92; 95\% CI, 3.70 to 9.45). Three studies were analyzed: one RCT (VPZ phase III) ${ }^{9}$ and two retrospective 
studies. ${ }^{26,27}$ Heterogeneity was very low $\left(\mathrm{I}^{2}=0 \%\right)$, indicating high reliability. In 2018, Li et al. ${ }^{12}$ published an MA of one RCT (eradication rate of VPZ vs PPI: $82.0 \%$ vs $40.0 \%$; OR, 6.83; 95\% CI, 3.63 to 12.86), and two retrospective studies (eradication rate: $80.8 \%$ vs $41.8 \%$; OR, 4.98 ; $95 \%$ CI, 2.47 to 10.03). We did not compare VPZ- and PPI-based therapies for CAM-resistant patients in our $\mathrm{RCT}^{25}$ for ethical reasons. PPI-based therapies are associated with poor eradication rates in subjects with CAM-resistant $H$. $p y$ lori, and such patients should receive VPZ-based therapy. We explored the utility of VPZ-based therapy for CAMresistant patients in a prospective study; the eradication rate was $82.9 \%$ (95\% CI, 67.9 to 92.8 ) ${ }^{25}$ thus in the range of "poor" (81\% to $84 \%){ }^{4}$ In 2020 , Suzuki et al. ${ }^{29}$ performed a prospective study (of the control arm of an RCT); the eradication rate was $76.2 \%$ (95\% CI, 60.5 to 87.9 ) in CAMresistant patients given VPZ-based therapy.

As we noted in "2.1. Mechanism of P-CAB action in patients with various lesions," the mechanism of this superiority is that rapid, strong, and stable acid block by $\mathrm{P}-\mathrm{CAB}$ results in AMPC and CAM becoming more effective, because at $\mathrm{pH}>5, \mathrm{H}$. pylori enters the growth phase. This is also supported by recent VPZ-AMPC dual therapy results.

In summary, VPZ-based therapy is superior to PPIbased therapy in patients with CAM-resistant $H$. pylori, but the eradication rate remains unacceptably low.

\section{CAM-susceptible subjects}

In 2017, Dong et al. ${ }^{10}$ published an MA evaluating firstline triple therapy consisting of AMPC and CAM in a CAM-susceptible subgroup; the eradication rate was $94.9 \%$ (95\% CI, 92.5 to 96.6 ) in the VPZ-based group versus $89.6 \%$ (95\% CI, 86.9 to 91.9 ) in the PPI-based group (OR, 2.02; $95 \% \mathrm{CI}, 1.23$ to 3.32 ). Four studies were analyzed: one RCT (VPZ phase III), ${ }^{9}$ one prospective study, ${ }^{24}$ and two retrospective studies. ${ }^{26,27}$ Heterogeneity was moderate $\left(I^{2}=45 \%\right)$, indicating moderate reliability. In 2018 , Li et al. ${ }^{12}$ published an MA based on five studies, consisting of one $\mathrm{RCT}^{25}$ plus the four studies evaluated by Dong et al. ${ }^{10}$ VPZ-based therapy was not superior to PPI-based therapy when the two RCTs were combined (eradication rate of VPZ vs PPI: $95.4 \%$ vs $92.8 \%$ ) or when the three non-RCTs were combined (eradication rate of VPZ vs PPI: $92.9 \%$ vs $86.2 \%$ ). The ORs were 1.63 ( $95 \% \mathrm{CI}, 0.74$ to $3.61 ; \mathrm{p}=0.225$ ) and 4.58 ( $95 \% \mathrm{CI}, 0.67$ to $31.45 ; \mathrm{p}=0.122$ ), respectively.

We performed an RCT to explore whether a clinically significant difference was apparent between VPZ-based and PPI-based triple therapies for CAM-susceptible $H$. pylori eradication. The eradication rates were $87.3 \%$ (95\% CI, 75.5 to 94.7) for VPZ-based therapy and 76.5\% (95\% CI, 62.5 to 87.2 ) for PPI-based therapy in the ITT analysis $(\mathrm{p}=0.21)$ and $88.9 \%(95 \% \mathrm{CI}, 77.4$ to 95.8$)$ and $86.7 \%(95 \%$ CI, 73.2 to 94.9$)$, respectively, in the PP analysis $(\mathrm{p}=0.77){ }^{25}$ There was no clinically significant difference.

Non-RCTs are at risk of several forms of bias that are lacking in RCTs. The differences between RCT and nonRCT analyses reflect these biases.

Rapid, strong, and stable acid block by $\mathrm{P}-\mathrm{CAB}$ results in AMPC and CAM becoming more effective, because at $\mathrm{pH}$ $>5, H$. pylori enters the growth phase. The major reason for VPZ-based superiority with CAM-resistant $H$. pylori is that AMPC works more effectively, as evidenced by the VPZ-AMPC dual therapy result. ${ }^{29}$ On the other hand, in a CAM-susceptible situation, PPI-induced acid suppression may be sufficient to be effective with the AMPC-CAM combination. The main reason for the failure of PPI-based or P-CAB-based eradication for CAM-susceptible H. pylori is based on the limit of the 7-day triple therapy regimen used in Japan. The results of VPZ-based therapy are not superior to those of PPI-based therapy, which shows that the limit of the 7-day triple therapy did not resolve the situation with $\mathrm{P}-\mathrm{CAB}$ use, and improvement of the administration frequency and dose of AMPC, and the treatment period of $\mathrm{P}-\mathrm{CAB}$-based triple therapy is necessary.

In summary, VPZ-based triple therapies are not superior to PPI-based SSTs in terms of eradicating CAMsusceptible $H$. pylori.

VPZ-COMPARED WITH PPI-BASED
SECOND-LINE TRIPLE THERAPY
CONSISTING OF AMPC AND MNZ

Two MAs of non-RCTs comparing 7-day triple therapy consisting of VPZ, AMPC, and MNZ with 7-day triple therapy consisting of a PPI, AMPC, and MNZ have appeared. In 2017, Dong et al. ${ }^{10}$ published a MA of non-RCTs and concluded that VPZ was not superior to PPIs when incorporated into a second-line therapy. In the ITT analysis, the eradication rates were $83.4 \%$ (95\% CI, 79.8 to 86.5 ) for VPZ-based therapy versus $81.2 \%$ (95\% CI, 79.5 to 83.5 ) for PPI-based therapy $(\mathrm{p}=0.79)$; in the PP analysis, the respective figures were $89.3 \%$ (95\% CI, 86.2 to 92.0 ) versus $90.1 \%$ (95\% CI, 88.3 to 91.6$)(\mathrm{p}=0.06) .{ }^{10}$ Six studies ${ }^{24,36,37,40,41,43}$ were evaluated. One study ${ }^{40}$ principally employed PPIbased triple therapy consisting of MNZ and STFX; 31 of total of 1,941 cases were reviewed in the MA. The heterogeneity was very low $\left(\mathrm{I}^{2}=0 \%\right)$, indicating that the MA was highly reliable.

In 2020, Shinozaki et al. ${ }^{52}$ published an MA of nonRCTs concluding that VPZ was superior to PPI when incorporated into second-line therapies. In the PP analysis, 
the eradication rates were $91.1 \%$ (95\% CI, 89.8 to 92.2 ) for VPZ-based therapy compared with $88.2 \%$ (95\% CI, 87.2 to 89.2 ) for PPI-based therapy $(\mathrm{p}<0.001)$. Sixteen studies $^{24,32,36,37,41,43,48,50,53-60}$ were evaluated. ${ }^{52}$ Heterogeneity was very low $\left(\mathrm{I}^{2}=0 \%\right)$, suggesting that the MA was very reliable. However, the latter seven studies ${ }^{54-60}$ are not listed in PubMed, indicating that they may be of poor quality and have not been critically apprised; none of those seven studies were included in several other MAs. ${ }^{10-12}$ After excluding those studies, the average eradication rates in the nine remaining studies were $90.9 \%$ (95\% CI, 89.6 to 92.1 ) for VPZ-based triple therapy and $88.3 \%$ (95\% CI, 87.2 to 89.3 ) for PPI-based triple therapy. The $2.6 \%$ difference lacks clinical significance. Also, most retrospective studies are at high risk of bias, lack pre-planned analyses, and used arbitrary numbers in the PPI-based arm that serve as historical controls. Another MA excluded most studies, considered "low-quality studies with poorly defined populations." ${ }^{12}$ In the MA, two propensity score-matched analyses ${ }^{52,53}$ were included. However, both works lacked antibiotic-resistance data, and this was not remedied by propensity score matching.

Several MAs seem to be very reliable in terms of low heterogeneity, but the conclusions differ. Publication bias may be in play, as retrospective studies with negative results may not be accepted by journals. Indeed, many positive results were published after one $\mathrm{MA} ;{ }^{55}$ one MA of retrospective studies published in $2020^{52}$ contained high numbers of subjects $(1,147 / 2,293$ cases of VPZ-based therapy and 2,251/3,854 cases of PPI-based therapy). Selection bias and confounding variables may be in play in other retrospective studies.

As shown in Table 2, that MA was almost entirely based on retrospective cohort trials and lacked data on antibiotic resistance; the groups were thus not matched in this context. Antibiotic-resistance data are essential when generalizing the results to countries or regions that vary in terms of the MNZ- or AMPC-resistance rate. In addition, the MA divided the patients into two groups based on VPZor PPI-based first-line therapy. Most studies do not do this; any assumption that the two groups are similar may be misleading. If first-line VPZ-based therapy is superior to PPI-based therapy, eradication is more difficult in those who fail first-line VPZ-based therapy. In summary, the finding of slight ( $2.6 \%)$ superiority of VPZ-based therapy was unreliable given the selection bias, confounding variables, and risk of publication bias, and the results are difficult to generalize because of a lack of antibiotic-resistance data.
VPZ- VERSUS PPI-BASED TRIPLE THERAPIES CONSISTING OF AMPC AND STFX FOR THIRD-LINE ERADICATION

In 2019, we reported an RCT comparing third-line VPZ- with PPI-based 7-day triple therapies consisting of AMPC after first-line triple therapy (AMPC and CAM) and second-line triple therapy (AMPC and MNZ) failures. ${ }^{61}$ The VPZ and AMPC doses were the same as those of the first- and second-line regimens; the STFX dose was $100 \mathrm{mg}$ bid (200 mg/day). The eradication rates were $75.8 \%$ (95\% CI, 57.7 to 88.9) for VPZ therapy versus 53.3\% (95\% CI, 34.3 to 71.7 ) for PPI therapy in the ITT analysis $(\mathrm{p}=0.071)$, and $83.3 \%$ (95\% CI, 65.3 to 94.4$)$ versus $57.1 \%$ (95\% CI, 37.2 to 75.5 ), respectively, in the PP analysis $(\mathrm{p}=0.043)$. In a retrospective study, Saito et al. ${ }^{32}$ reported eradication rates of $93.0 \%$ (95\% CI, 83.0 to 98.1) for VPZ therapy versus $54.2 \%$ (95\% CI, 32.8 to 74.4 ) for PPI therapy (esomeprazole) in the ITT analysis $(\mathrm{p}<0.001)$, and $93.0 \%$ (95\% CI, 83.0 to 98.1 ) versus $56.5 \%$ (95\% CI, 34.5 to 76.8 ), respectively, in the $\mathrm{PP}$ analysis $(\mathrm{p}<0.001)$. In summary, a third-line VPZ-based triple therapy consisting of AMPC and STFX is more effective than a PPI-based regimen, but a confirmatory RCT is required.

\section{VPZ- VERSUS PPI-BASED TRIPLE THERAPIES INVOLVING CAM AND MNZ}

In 2017, Ono et al. ${ }^{40}$ published a retrospective study comparing 7-day triple therapy consisting of VPZ, CAM, and MNZ with 7-day triple therapy consisting of PPI, CAM, and MNZ. The VPZ-based regimen was associated with a higher eradication rate than that of the PPI-based treatment, thus $92.9 \%(n=14)$ versus $46.2 \%(n=13)$ in the ITT analysis $(p=0.0128)$ and $92.9 \%$ versus $54.6 \%$ in the PP analysis. In 2017, we reported a registered, prospective, non-randomized study comparing VPZ-based and PPIbased regimens, as mentioned above. The eradication rate was $100 \%$ (95\% CI, 86.1 to 100) for the VPZ-based therapy versus $83.3 \%$ (95\% CI, 65.3 to 94.4) for the PPI-based therapy in the ITT analysis, and $100 \%$ (95\% CI, 86.1 to 100$)$ versus $82.7 \%$ (95\% CI, 64.2 to 94.2 ), respectively, in the PP analysis. ${ }^{62}$ Thus, VPZ-based triple therapy involving CAM and MNZ seems to be superior to PPI-based therapy. In summary, a VPZ-based triple therapy (CAM and MNZ) may be better than a PPI-based regimen, but both studies were non-RCTs and lacked data on antibiotic resistance. 


\section{CONCLUSIONS AND FUTURE DIRECTIONS}

Any attempt to answer the question "Is $\mathrm{P}-\mathrm{CAB}$ really superior to a PPI in terms of $H$. pylori eradication?" is limited by the setting in which we work. VPZ (a P-CAB) was used in 7-day triple therapies at the dose covered by the Japanese national insurance system. The CAM-resistance rate was approximately $33 \%$, whereas the MNZ-resistance rate was low $(<5 \%)$ and the AMPC-resistance rate was very low. Tegoprazan and other P-CABs should be trialed in terms of $H$. pylori eradication in the future. In addition, the study was performed mainly in Japan. Diet and human genetics are also influence the stomach $\mathrm{pH}$. Thus, further studies outside Japan are needed to generalize the result to global populations.

An MA of RCTs comparing VPZ- and PPI-based firstline triple therapies consisting of AMPC and CAM may be generalizable to populations comprising approximately $30 \%$ of CAM-resistant subjects, but reliability is poor because of high heterogeneity and a risk of selection bias (poor allocation concealment). First-line VPZ-based triple therapy involving AMPC and CAM are superior to PPIbased regimens in patients with CAM-resistant $H$. pylori, but the eradication rate remains unacceptably low. Firstline VPZ-based triple therapies consisting of AMPC and CAM are not superior to PPI-based regimens in patients with CAM-susceptible $H$. pylori, as revealed by two RCTs. The slightly $(\sim 2.6 \%)$ higher success rate of second-line VPZ-based triple therapy compared with PPI-based triple therapy (AMPC and MNZ) is unreliable given the selection bias, confounding variables and risk of publication bias, and it is difficult to generalize the results because of the lack of antibiotic-resistance information. Further RCTs are required. Third-line VPZ-based triple therapies involving AMPC and STFX may be more effective than PPIbased regimens, but a confirmatory RCT is required. VPZbased triple therapies involving CAM and MNZ may be better than PPI-based regimens, but only non-RCT data are available, and information on antibiotic resistance is lacking. Finally, more RCTs with antibiotic-resistance data are required in populations outside Japan if P-CABs are to replace PPIs worldwide.

\section{CONFLICTS OF INTEREST}

No potential conflict of interest relevant to this article was reported.

\section{ACKNOWLEDGEMENTS}

We thank Eijin Hashimoto (Yokohama City University School of Medicine, Yokohama, Japan) for the assistance with preparation of the tables.

\section{ORCID}

Soichiro Sue https://orcid.org/0000-0002-4645-1291

Shin Maeda https://orcid.org/0000-0002-0246-1594

\section{REFERENCES}

1. Sue S, Shibata W, Maeda S. Helicobacter pylori-induced signaling pathways contribute to intestinal metaplasia and gastric carcinogenesis. Biomed Res Int 2015;2015:737621.

2. Ford AC, Yuan Y, Moayyedi P. Helicobacter pylori eradication therapy to prevent gastric cancer: systematic review and meta-analysis. Gut 2020;69:2113-2121.

3. Malfertheiner P, Megraud F, O'Morain CA, et al. Management of Helicobacter pylori infection: the Maastricht V/ Florence Consensus Report. Gut 2017;66:6-30.

4. Graham DY, Lu H, Yamaoka Y. A report card to grade Helicobacter pylori therapy. Helicobacter 2007;12:275-278.

5. Labenz J. Current role of acid suppressants in Helicobacter pylori eradication therapy. Best Pract Res Clin Gastroenterol 2001;15:413-431.

6. Villoria A, Garcia P, Calvet X, Gisbert JP, Vergara M. Metaanalysis: high-dose proton pump inhibitors vs. standard dose in triple therapy for Helicobacter pylori eradication. Aliment Pharmacol Ther 2008;28:868-877.

7. Vallve M, Vergara M, Gisbert JP, Calvet X. Single vs. double dose of a proton pump inhibitor in triple therapy for Helicobacter pylori eradication: a meta-analysis. Aliment Pharmacol Ther 2002;16:1149-1156.

8. Huang J, Hunt RH. The importance of clarithromycin dose in the management of Helicobacter pylori infection: a metaanalysis of triple therapies with a proton pump inhibitor, clarithromycin and amoxycillin or metronidazole. Aliment Pharmacol Ther 1999;13:719-729.

9. Murakami K, Sakurai Y, Shiino M, Funao N, Nishimura A, Asaka M. Vonoprazan, a novel potassium-competitive acid blocker, as a component of first-line and second-line triple therapy for Helicobacter pylori eradication: a phase III, randomised, double-blind study. Gut 2016;65:1439-1446.

10. Dong SQ, Singh TP, Wei X, Yao H, Wang HL. Review: a Japanese population-based meta-analysis of vonoprazan versus PPI for Helicobacter pylori eradication therapy: is superiority an illusion? Helicobacter 2017;22:e12438. 
11. Jung YS, Kim EH, Park CH. Systematic review with metaanalysis: the efficacy of vonoprazan-based triple therapy on Helicobacter pylori eradication. Aliment Pharmacol Ther 2017;46:106-114.

12. Li M, Oshima T, Horikawa T, et al. Systematic review with meta-analysis: vonoprazan, a potent acid blocker, is superior to proton-pump inhibitors for eradication of clarithromycin-resistant strains of Helicobacter pylori. Helicobacter 2018;23:e12495

13. Lyu QJ, Pu QH, Zhong XF, Zhang J. Efficacy and safety of vonoprazan-based versus proton pump inhibitor-based triple therapy for Helicobacter pylori eradication: a metaanalysis of randomized clinical trials. Biomed Res Int 2019;2019:9781212.

14. Jenkins H, Sakurai Y, Nishimura A, et al. Randomised clinical trial: safety, tolerability, pharmacokinetics and pharmacodynamics of repeated doses of TAK-438 (vonoprazan), a novel potassium-competitive acid blocker, in healthy male subjects. Aliment Pharmacol Ther 2015;41:636-648.

15. Kagami T, Sahara S, Ichikawa H, et al. Potent acid inhibition by vonoprazan in comparison with esomeprazole, with reference to CYP2C19 genotype. Aliment Pharmacol Ther 2016;43:1048-1059.

16. Yamasaki H, Kawaguchi N, Nonaka M, et al. In vitro metabolism of TAK-438, vonoprazan fumarate, a novel potassiumcompetitive acid blocker. Xenobiotica 2017;47:1027-1034.

17. Sachs G, Meyer-Rosberg K, Scott DR, Melchers K. Acid, protons and Helicobacter pylori. Yale J Biol Med 1996;69:301316.

18. Sugano K. Vonoprazan fumarate, a novel potassium-competitive acid blocker, in the management of gastroesophageal reflux disease: safety and clinical evidence to date. Therap Adv Gastroenterol 2018;11:1756283X17745776.

19. Kim YG, Jang BI, Kim TN. A matched case-control study of a novel acid-pump antagonist and proton-pump inhibitor for the treatment of iatrogenic ulcers caused by endoscopic submucosal dissection. Gut Liver 2010;4:25-30.

20. Cho YK, Choi MG, Choi SC, et al. Randomised clinical trial: tegoprazan, a novel potassium-competitive acid blocker, or lansoprazole in the treatment of gastric ulcer. Aliment Pharmacol Ther 2020;52:789-797.

21. Lee KJ, Son BK, Kim GH, et al. Randomised phase 3 trial: tegoprazan, a novel potassium-competitive acid blocker, vs. esomeprazole in patients with erosive oesophagitis. Aliment Pharmacol Ther 2019;49:864-872.

22. Dore MP, Leandro G, Realdi G, Sepulveda AR, Graham DY. Effect of pretreatment antibiotic resistance to metronidazole and clarithromycin on outcome of Helicobacter pylori therapy: a meta-analytical approach. Dig Dis Sci 2000;45:6876.

23. Horie R, Handa O, Ando T, et al. Helicobacter pylori eradi- cation therapy outcome according to clarithromycin susceptibility testing in Japan. Helicobacter 2020;25:e12698.

24. Sue S, Kuwashima H, Iwata Y, et al. The superiority of vonoprazan-based first-line triple therapy with clarithromycin: a prospective multi-center cohort study on Helicobacter pylori eradication. Intern Med 2017;56:1277-1285.

25. Sue S, Ogushi M, Arima I, et al. Vonoprazan- vs protonpump inhibitor-based first-line 7-day triple therapy for clarithromycin-susceptible Helicobacter pylori: a multicenter, prospective, randomized trial. Helicobacter 2018;23:e12456.

26. Noda H, Noguchi S, Yoshimine T, et al. A novel potassiumcompetitive acid blocker improves the efficacy of clarithromycin-containing 7-day triple therapy against Helicobacter pylori. J Gastrointestin Liver Dis 2016;25:283-288.

27. Matsumoto H, Shiotani A, Katsumata R, et al. Helicobacter pylori eradication with proton pump inhibitors or potassium-competitive acid blockers: the effect of clarithromycin resistance. Dig Dis Sci 2016;61:3215-3220.

28. Shinmura T, Adachi K, Yamaguchi Y, et al. Vonoprazanbased triple-therapy could improve efficacy of the tailored therapy of Helicobacter pylori infection. J Gastrointestin Liver Dis 2019;28:389-395.

29. Suzuki S, Gotoda T, Kusano C, et al. Seven-day vonoprazan and low-dose amoxicillin dual therapy as first-line Helicobacter pylori treatment: a multicentre randomised trial in Japan. Gut 2020;69:1019-1026.

30. Sugimoto M, Ban H, Hira D, et al. Letter: CYP3A4/5 genotype status and outcome of vonoprazan-containing Helicobacter pylori eradication therapy in Japan. Aliment Pharmacol Ther 2017;45:1009-1010.

31. Tanabe H, Yoshino K, Ando K, et al. Vonoprazan-based triple therapy is non-inferior to susceptibility-guided proton pump inhibitor-based triple therapy for Helicobacter pylori eradication. Ann Clin Microbiol Antimicrob 2018;17:29.

32. Saito Y, Konno K, Sato M, et al. Vonoprazan-based third-line therapy has a higher eradication rate against sitafloxacinresistant Helicobacter pylori. Cancers (Basel) 2019;11:116.

33. Suzuki S, Gotoda T, Kusano C, Iwatsuka K, Moriyama M. The efficacy and tolerability of a triple therapy containing a potassium-competitive acid blocker compared with a 7-day PPI-based low-dose clarithromycin triple therapy. Am J Gastroenterol 2016;111:949-956.

34. Shinozaki S, Nomoto H, Kondo Y, et al. Comparison of vonoprazan and proton pump inhibitors for eradication of Helicobacter pylori. Kaohsiung J Med Sci 2016;32:255-260.

35. Shichijo S, Hirata Y, Niikura R, et al. Vonoprazan versus conventional proton pump inhibitor-based triple therapy as first-line treatment against Helicobacter pylori: a multicenter retrospective study in clinical practice. J Dig Dis 2016;17:670-675.

36. Yamada S, Kawakami T, Nakatsugawa Y, et al. Usefulness of 
vonoprazan, a potassium ion-competitive acid blocker, for primary eradication of Helicobacter pylori. World J Gastrointest Pharmacol Ther 2016;7:550-555.

37. Tsujimae M, Yamashita H, Hashimura H, et al. A comparative study of a new class of gastric acid suppressant agent named vonoparazan versus esomeprazole for the eradication of Helicobacter pylori. Digestion 2016;94:240-246.

38. Katayama Y, Toyoda K, Kusano Y, et al. Efficacy of vonoprazan-based second-line Helicobacter pylori eradication therapy in patients for whom vonoprazan-based first-line treatment failed. Gut 2017;66:752-753.

39. Kajihara Y, Shimoyama T, Mizuki I. Analysis of the cost-effectiveness of using vonoprazan-amoxicillin-clarithromycin triple therapy for first-line Helicobacter pylori eradication. Scand J Gastroenterol 2017;52:238-241.

40. Ono S, Kato M, Nakagawa S, Mabe K, Sakamoto N. Vonoprazan improves the efficacy of Helicobacter pylori eradication therapy with a regimen consisting of clarithromycin and metronidazole in patients allergic to penicillin. Helicobacter 2017;22:e12374.

41. Sakurai K, Suda H, Ido Y, et al. Comparative study: vonoprazan and proton pump inhibitors in Helicobacter pylori eradication therapy. World J Gastroenterol 2017;23:668-675.

42. Maruyama M, Tanaka N, Kubota D, et al. Vonoprazan-based regimen is more useful than PPI-based one as a first-line Helicobacter pylori eradication: a randomized controlled trial. Can J Gastroenterol Hepatol 2017;2017:4385161.

43. Nishizawa T, Suzuki H, Fujimoto A, et al. Effects of patient age and choice of antisecretory agent on success of eradication therapy for Helicobacter pylori infection. J Clin Biochem Nutr 2017;60:208-210.

44. Tanabe H, Ando K, Sato K, et al. Efficacy of vonoprazanbased triple therapy for Helicobacter pylori eradication: a multicenter study and a review of the literature. Dig Dis Sci 2017;62:3069-3076.

45. Ozaki H, Harada S, Takeuchi T, et al. Vonoprazan, a novel potassium-competitive acid blocker, should be used for the Helicobacter pylori eradication therapy as first choice: a large sample study of vonoprazan in real world compared with our randomized control trial using second-generation proton pump inhibitors for Helicobacter pylori eradication therapy. Digestion 2018;97:212-218.

46. Mori N, Nishiura Y, Suga D, et al. Second-line triple therapy in failures with vonoprazan-based triple therapy for eradication of Helicobacter pylori. Biomed Rep 2018;9:169-174.

47. Shinozaki S, Osawa H, Sakamoto H, et al. Pre-treatment with proton pump inhibitors decreases the success of primary Helicobacter pylori eradication using a vonoprazanbased regimen. Kaohsiung J Med Sci 2018;34:456-460.

48. Kusunoki M, Yuki M, Ishitobi H, et al. Effect of age on effectiveness of vonoprazan in triple therapy for Helicobacter pylori eradication. Intern Med 2019;58:1549-1555.
49. Nishida T, Tsujii Y, Okamoto A, et al. A triple-drug blisterpackaged drug with vonoprazan improves first-line eradication of Helicobacter pylori in elderly patients: a retrospective propensity score-matched cohort study. Digestion 2020;101:608-614.

50. Mori H, Suzuki H, Omata F, et al. Current status of firstand second-line Helicobacter pylori eradication therapy in the metropolitan area: a multicenter study with a large number of patients. Therap Adv Gastroenterol 2019;12:1756284819858511.

51. Furuta T, Yamade M, Kagami T, et al. Dual therapy with vonoprazan and amoxicillin is as effective as triple therapy with vonoprazan, amoxicillin and clarithromycin for eradication of Helicobacter pylori. Digestion 2020;101:743-751.

52. Shinozaki S, Kobayashi Y, Osawa H, et al. Effectiveness and safety of vonoprazan versus proton pump inhibitors for second-line Helicobacter pylori eradication therapy: systematic review and meta-analysis. Digestion 2021;102:319-325.

53. Nabeta $\mathrm{H}$, Shinozaki S, Abe Y, et al. A potassium-competitive acid blocker-based regimen as second-line therapy improves Helicobacter pylori eradication. Digestion 2020;101:332-338.

54. Murai R, Hada T. Helicobacter pylori eradication therapy using vonoprazan-based triple regimen: report of $1039 \mathrm{pa}-$ tients. Tokyoto Ishikai Zasshi 2017;70:73-77.

55. Mukai R, Handa O, Katada K, et al. Retrospective analysis of Helicobacter pylori eradication therapy classified by age. Ulcer Res 2017;(44):80-84.

56. Ishihara R. Helicobacter pylori eradication therapy using vonoprazan-based regimen. J Jpn Health Med Assoc 2017;5:2831.

57. Yoshizumi Y, Seo M, Takei R, Udagawa K, Yoshizumi H, Nagata M. Eradication therapy of Helicobacter pylori. Saitamaken Igakkai Zasshi 2016;51:66-71.

58. Saegusa Y, Mihara S, Sato A, et al. Helicobacter pylori eradication therapy with vonoprazan-based regien: comparison with past regimens. Helicobacter Res 2016;20:85-88.

59. Yoshida H, Kurita S, Ichimatsu O, Takabatake K. Eradication therapy of Helicobacter pylori in eiju general hospital. J Res Institute Life Ext 2016;(28):22-28.

60. Sato Y, Takenaka R, Ishikawa H, et al. Usefulness of vonoprazan-based triple therapy for eradication of Helicobacter pylori in northern okayama. Tsuyama Chuobyouin Igaku Zasshi 2017;31:3-10.

61. Sue S, Shibata W, Sasaki T, et al. Randomized trial of vonoprazan-based versus proton-pump inhibitor-based thirdline triple therapy with sitafloxacin for Helicobacter pylori. J Gastroenterol Hepatol 2019;34:686-692.

62. Sue S, Suzuki N, Shibata W, et al. First-line Helicobacter pylori eradication with vonoprazan, clarithromycin, and metronidazole in patients allergic to penicillin. Gastroenterol Res Pract 2017;2017:2019802. 